\title{
Letter: faecal volatile organic metabolites, promising biomarkers in inflammatory bowel disease and Letter: faecal volatile organic metabolites as novel diagnostic biomarkers in inflammatory bowel disease. Authors' reply
}

\author{
I. Ahmed*, R. Greenwood† , B. Costelloł , N. Ratcliffeł \& C. Probert§ \\ *Department of Gastroenterology, University Hospital Southampton, Southampton, UK. \\ † Department of Research and Development, Bristol Royal Infirmary, Bristol, UK. \\ ¥ Institute of Biosensing Technology, University of the West of England, Bristol, UK. \\ $\S$ Gastroenterology Research Unit, Institute of Translational Medicine, University of Liverpool, \\ Liverpool, UK. \\ E-mail: norman.ratcliffe@uwe.ac.uk
}

doi:10.1111/apt.13617

SIR, We read with great interest the very valuable comments by both Furnari et al., 1 and Srinavas, 2 regarding our recently published paper on faecal volatile metabolite in inflammatory bowel disease. 3 In this study, we investigated the changes in faecal metabolites in different subsets of patients with Crohn's disease and ulcerative colitis in comparison with healthy controls.

We agree with the comments by Furnari et al. about the lack of detailed dietary data about the study participants, which could have some potential effect on the changes observed in the faecal metabolites. This is a very important question and we had already highlighted this weakness of our study in the discussion section. Dietary variations can potentially affect the faecal metabolites through their effects predominantly on gut microbial dysbiosis.4, 5 Patients with inflammatory bowel disease change their diet often in response to their symptoms, which makes it difficult (although not impossible) to collect very accurate dietary data. Such dietary comparison would have been easier and valuable in a precisely controlled setting - for example, in patients on elemental diet. None of the participants in this study is on elemental diet and due to the lack of detailed dietary data; the study was unable to shed any light on the effects of diet on the faecal metabolites. However, considering the high importance of diet, we are planning future studies to investigate any effect of diet on the faecal metabolites in IBD.

We are in agreement by the commentary offered by Srivanas regarding the influence of medication on the faecal metabolites. Detailed information about the medicines that the study participants were on was provided and the patients were continued on their existing medications. Our study did not detect any active or inactive metabolites of these medicines in the faeces of study participants. Although no solid conclusion could be drawn about the effect of medications on faecal metabolites with the current data but such an important question should be considered in future studies by including patients before and after starting drug treatment.

Our study detected a number of faecal metabolites such as heptanal, 1-octen-3-ol, 2-piperidinone and 6- methyl-2-heptanone, methanethiol, 3-methylphenol, short-chain fatty acids and ester 
derivatives that could be discriminatory in separating different groups of IBD. As pointed out by Srinavas et al., it would be ideal to find a single diagnostic metabolite in IBD which could provide information about the activity, type and extent of disease; however, the discrimination between the subtype of our study groups was shown by using a discriminatory model based on a number of metabolites. We discussed the important of each class (aldehyde, alcohol and ketones) of these discriminatory metabolites and its possible correlation with the gut microbial dysbiosis. With the development of more sophisticated analytical techniques, future research may be able to discover single diagnostic biomarker in IBD.

Our study groups comprised of patients with different extent, type and activity of the disease but we did not have any data regarding severity and duration of the disease. We believe this would be the next step in future studies to investigate the changes in faecal metabolites of patient at the initial diagnosis prior to commencing any medication, and in patients with different duration and severity of the disease.

\section{ACKNOWLEDGEMENT}

The authors' declarations of personal and financial interests are unchanged from those in the original article. 3

\section{REFERENCES}

1. Furnari M, Bodini G, Edoardo GG, Savarino V. Letter: faecal volatile organic metabolites, promising biomarkers in inflammatory bowel disease. Aliment Pharmacol Ther 2016; 43: 1240-1.

2. Srinivas N. Letter: faecal volatile organic metabolites as novel diagnostic biomarkers in inflammatory bowel disease. Aliment Pharmacol Ther 2016; 43: 1239-40.

3. Ahmed I, Greenwood R, Costello B, Ratcliffe N, Probert CS. Investigation of faecal volatile organic metabolites as novel diagnostic biomarkers in inflammatory bowel disease. Aliment Pharmacol Ther 2016; 43: 596-611.

4. Turnbaugh PJ, Ridaura VK, Faith JJ, et al. The effect of diet on the human gut microbiome: a metagenomic analysis in humanized gnotobiotic mice. Sci Transl Med 2009; 1: 6-14.

5. Zimmer J, Lange B, Frick JS, et al. A vegan or vegetarian diet substantially alters the human colonic faecal microbiota. Eur J Clin Nutr 2012; 66: 53-60. 